\title{
Celiac disease and dental enamel defects: what is the link?
}

\author{
Raffaella Mormile $^{1}$
}

Accepted: 30 September 2015 / Published online: 6 October 2015

(C) Springer-Verlag Berlin Heidelberg 2015

\section{Dear Editor:}

Celiac disease is a lifelong autoimmune condition caused by intolerance to the dietary gluten in genetically predisposed individuals resulting in villous atrophy, inflammation, and crypt cell hyperplasia in the proximal small intestine. The detection of gluten-reactive CD4 $+\mathrm{T}$ cells producing interferon- $\gamma($ IFN- $\gamma)$ in the small-intestine mucosa of CD patients implies a T helper type 1 (Th1) polarization. The activation of an aberrant Th1 immune reaction initiated by the presence of gluten is a crucial event in the onset of $\mathrm{CD}$. IFN- $\gamma$ is considered a master regulator of the immunopathology in CD. Functional variants of IFN- $\gamma$ gene have been associated with $\mathrm{CD}$ susceptibility. It has been demonstrated that the Janus kinase-signal transducer and activator of transcription (JAK-Stat) pathway is implicated in the pathogenesis of CD. The JAK-Stat pathway plays an important role in regulating the immune response. The JAK-Stat pathway seems to mediate the early Th1 IFN- $\gamma$-directed response by activating genes transcription through different Stat proteins. Interestingly, IFN- $\gamma$ is described as a potent activator of the JAK-Stat pathway. It has been speculated that IFN- $\gamma$ may contribute to the maintenance of the chronic intestinal inflammation through the JAK-Stat pathway by regulating the expression and the activation of Stat-1. It has been observed that Stat-1 is an important feature of the inflammatory response in CD. Overproduction of IFN- $\gamma$ has been shown to be invariably

Raffaella Mormile

raffaellamormile@alice.it

1 Division of Pediatrics and Neonatology, Moscati Hospital, Via A. Gramsci, 3, 81031 Aversa, Italy related to high Stat-1 activation in CD patients. CD exhibits a spectrum of clinical and pathologic manifestations. Although CD patients may suffer primarily from gastrointestinal symptoms, any organ system may be involved in $\mathrm{CD}$ including permanent teeth. It has been reported that developmental defects of the dental enamel are more frequently detected in patients with $\mathrm{CD}$ in comparison to healthy people. Celiac-type dental enamel defects are characterized by symmetry, chronology, and occurrence with the same group of teeth. Dental enamel hypoplasia is defined as an autoimmune response of the enamel organ. The cause of the particular enamel mineralization defect in $\mathrm{CD}$ is yet to be determined. The role of nutritional, genetic, and immunological factor has been taken into consideration. Dental enamel defects correlate with the period of interruption of amelogenesis. Stat1 signaling has been shown to be a key player in amelogenesis and dentinogenesis in rats. It has been highlighted that IFN- $\gamma$-mediated activation of Stat-1 may be involved in the events leading directly to early enamel formation. It has been observed that Stat-1 gain-of-function mutation may cause dental enamel abnormalities. All these contentions led us to hypothesize that the disruption of dental enamel development in CD may be due to a gain of function mutation in Stat-1 by IFN- $\gamma$ gene polymorphisms. Research studies are required in order to determine whether IFN- $\gamma /$ Stat-1 signaling pathway may be responsible for other extra-intestinal manifestations of CD. Assessment of dental enamel defects might be utilized as a valuable tool in monitoring adherence to a gluten-free diet and identifying slight dietary transgressions and/or consumption of certain amounts of gluten unconsciously due to contamination of gluten-free products. 